\title{
Peligros de la modificación de conducta en el tratamiento de la anorexia nerviosa $*$.
}

\section{Hilde Bruch, M. D.}

En los últimos años se ha señalado, de una forma excesiva, la eficacia de la modificación de conducta en el tratamiento de la anorexia nerviosa. Se ha supuesto, de forma errónea, que la imposición de una mejoría de la emaciación, el síntoma más llamativo, es un tratamiento adecuado para este trastorno tan grave y complejo. Los resultados que se han publicado adolecen de falta de seguimiento.

En estos pacientes, este método de tratamientos les llevó a un empeoramiento de su estado. A pesar de conseguir un aumento de peso pasajero, la modificación de conducta debe considerarse como potencialmente dañina en la anorexia nerviosa. (Jama, 230: 1419-1422, 1974.)

La anorexia nerviosa ha sido considerada desde siempre como una enfermedad grave con grandes dificultades de tratamiento. La comprensión de la dinámica subyacente en un tratamiento psicoterapéutico ha producido curaciones a largo plazo (1-3, p. 334). En contraste con este tratamiento, que es complejo e individualizado, se ha afirmado con demasiado optimismo la existencia de un procedimiento nuevo y sencillo, la modificación de conducta, por medio del cual se recompensa el incremento de peso, utilizando como "refuerzos positivos» la posibilidad de realizar actividades deseables (4-6).

El entusiasmo por este nuevo enfoque se demuestra en el hecho de que esta revista le dedicó dos editoriales en que apoyaba la eficacia del método (7-8). En una carta al editor, Feinstein planteó los posibles peligros de este enfoque simplista. También señaló que los resultados se habían publicado sin estudios de seguimiento. Yo estoy en condiciones de aportar información sobre los efectos perjudiciales de los pacientes tratados con este método. Sus tristes historias ilustran que los métodos de modificación de conducta no son sólo peligrosos en potencia, sino en la realidad.

\section{OBSERVACIONES PERSONALES}

Durante los tres últimos años, nos fue solicitada consulta por más de cincuenta pacientes con anorexia nerviosa refractaria al tratamiento. Muchas veces se mencionaba la modificación de conducta como el método de tratamiento utilizado, por medio del cual a la ganancia de

* "Perils of behavior modification intreatment of anorexia nervosa", Jama, 1974, vol. 230-10, páginas 1419-1422. Reproducido con autorización. ${ }^{\circ}$ De esta traducción Estudios de Psicologia. 
peso inicial le había seguido una pérdida de peso posterior, y un deterioro del cuadro general. Estos pacientes, que venían de todas partes del pais, nos indicaron que la modificación de conducta se utilizaba profusamente.

A los pacientes y familiares se les hace una evaluación completa sólo cuando lo pide el médico que envia al paciente. Se obtiene información escrita de todos los intentos de tratamiento previo, pidiéndola a pacientes, familiares, médicos y hospitales. Lo más frecuente es encontrar una información de mejoría de estas pacientes al alta. Posteriormente comienza el deterioro. En ningún caso se encontró una contradicción entre los informes del hospital y la información subjetiva de los pacientes sobre la forma de tratamiento. Desde 1972 fueron estudiados nueve pacientes con los que se había utilizado la modificación de conducta con resultados perjudiciales. Numerosas cartas nos han contado historias similares.

Para ilustrar este articulo he escogido tres pacientes vistas hace poco (a principios de 1974). Eran de distinta raza y religión, y venian de distintas partes del país (área metropolitana de N. Y., de una ciudad industrial del Sur, y del Midwest).

\section{INFORME DE LOS CASOS}

\section{Caso 1.}

Una joven de diecinueve años vino a consulta en enero de 1974, después de haber estado anorética y amenorréica desde hacía $31 / 2$ años. Pesaba $32,5 \mathrm{~kg}$. y media $1,60 \mathrm{~m}$. de alto. Durante su infancia había tenido un peso normal, y su peso más alto, a los dieciseis años, había sido de $53 \mathrm{~kg}$.

En esa época sus padres se divorciaron, y durante ese periodo de tensión se aisló de sus amistades y perdió peso rápidamente. A pesar de esto, acabó el "High school" con notas superiores y completó dos trimestres del "College». En aquellos momentos su peso habia ba- jado hasta $35 \mathrm{~kg}$. y fue admitida en el Servicio Psiquiátrico del Hospital Universitario, donde fue incluida en un programa de modificación de conducta. Este incluia deprivación de todas las actividades y contactos con los demás basta que adquiriera la ganancia de peso prescrita. A los dos meses su peso se elevó a los $43 \mathrm{~kg}$., pero ella fue deprimiéndose poco a poco y se escapó del hospital, alquiló un coche y condujo hacia su casa a una velocidad vertiginosa, con intento suicida. Continuó deprimida y con pensamientos suicidas. Perdió el peso impuesto reanudando un régimen estricto.

Durante la consulta se mostraba profundamente deprimida y tenía pánico de ser hospitalizada mediante engaños para conseguir una ganancia de peso en contra de su voluntad. Este miedo dificultó la respuesta a la psicoterapia posterior. Sólo después de haberse establecido una relación significativa fue capaz de obtener peso sin deprimirse. En este momento su peso es casi de $44 \mathrm{~kg}$.

\section{Caso 2.}

Esta paciente tenia diecisiete años $y$ medio en la fecha de la consulta, en marzo de 1974; había estado anorética durante dos años. Pesaba $32,5 \mathrm{~kg}$. y medía $1,65 \mathrm{~m}$. de alto. Durante la infancia había disfrutado de una salud excelente, y había estado un poco gordita a los oncedoce años. En esa época aparecieron los caracteres sexuales secundarios, a pesar de no haber tenido la menstruación. A los doce años su peso era de $46 \mathrm{~kg}$. Durante una enfermedad, perdió peso hasta aproximadamente $41,5 \mathrm{~kg}$., le gustó su aspecto más delgado, y desde entonces (1968) controló cuidadosamente su peso, manteniéndolo celosamente en $43,2 \mathrm{~kg}$. Durante estos años creció de 1,55 a $1,65 \mathrm{~m}$.

La anorexia se manifestó dramáticamente durante la primavera de 1972, cuando tenía quince años. Llegó a tener un miedo mórbido a engordar, caminaba sin parar, haciendo ejercicio compulsivamente y dormía muy poco. En diciem- 
bre de 1972 , pesando $33,5 \mathrm{~kg}$. fue admitida en un hospital de Nueva York, famoso por sus experiencias con anorexia nerviosa. Perdió algo más de peso, hasta $31 \mathrm{~kg}$. Se estableció un tratamiento de modificación de conducta. Se le exigió aislamiento, estancia en cama, deprivación de todas las actividades, no acceso a la radio, libros o habilidades artísticas, excepto cuando hubiera obtenido las cantidades de peso prescritas. Su peso aumentó de 31 a $42,5 \mathrm{~kg}$. en menos de dos meses. Se le dió el alta en un estado altamente mejorado, a pesar de que ella se sentía desdichada con su unuevo cuerpo gordom. Sentía que había perdido todo control sobre su alimentación y comenzó a dedicarse a incontrolados episodios de bulimia, seguidos de vómitos provocados.

Como su peso descendió a 39,5 kg., en menos de dos semanas, fue enviada a la unidad de psiquiatria de un hospital universitario, en un estado apático que también se experimenta en la anorexia nerviosa. Allí se la incluyó en otro programa de modificación de conducta, por medio del cual la alimentación era reforzada con la amenaza de tres tubos alimenticios en el día en que fallara en obtener el peso prescrito.

Su peso alcanzó los $47 \mathrm{~kg}$. en tres meses; fue dada de alta en un estado muy mejorado, a pesar de estar profundamente deprimida, incluso con pensamientos suicidas. Se sintió humillada por el procedimiento, y sintió que se le había coaccionado a rellenarse contra su voluntad. Después de ser dada de alta, fue seguida regularmente por un médico, para el que ella conseguía su peso requerido de $40 \mathrm{~kg}$. por medio de un comer descontrolado antes de ir a su consulta. Continuó su trabajo escolar con resultados excepcionales y realizó muchas otras actividades.

Fue la paciente quien pidió a sus padres una consulta con la esperanza de encontrar ayuda para su angustia mental. Pero perdió su peso hasta $32,5 \mathrm{~kg}$. por si acaso fuera de nuevo introducida, por medio del engaño, en un plan de obten- ción de peso. Cuando reconoció la filosofia diferente de nuestro acercamiento terapéutico, ganó peso hasta $37 \mathrm{~kg}$. en tres semanas, porque aceptó la exposición objetiva de que no podría realizarse ningún trabajo terapéutico significativo mientras estuviera en un estado de emaciación. Volvió a su casa para graduarse en la High School. Varios compañeros de clase han hecho comentarios sobre el cambio de su actitud y apariencia. Tiene planeado asistir a la Universidad en una comunidad con excelentes facilidades para realizar una psicoterapia continuada.

\section{Caso 3.}

La paciente tenía veinte años, pesaba $35 \mathrm{~kg}$. y medía $1,52 \mathrm{~m}$. cuando vino a consulta en junio de 1974. Había estado en tratamiento por anorexia nerviosa durante los siete años anteriores, y tenía en ese momento alteraciones graves del sueño, alimentación compulsiva y vómitos. Había sido alta cuando era pequeña y envidiaba a los niños que eran más bajos y más delgados. La anorexia comenzó de modo poco llamativo cuando tenía trece años, pesaba $42,5 \mathrm{~kg}$. y medía $1,51 \mathrm{~m}$. Cuando en clase de alimentación se pidió a los alumnos que escribieran todo lo que comían, se sintió avergonzada de lo que ella comía y decidió restringir la cantidad de comida, y ha estado preocupada con ello desde entonces. En este momento aún no ha tenido la menarquia.

Tuvo una considerable pérdida de peso, hasta $32,5 \mathrm{~kg}$., se observó también que la paciente se volvió menos comunicativa y más introvertida. La llevaron a un psicoanalista para que la tratara y fue admitida en un servicio psiquiátrico infantil donde ganó algo de peso, aproximadamente llegó a $36 \mathrm{~kg}$. Después de pasado un año en casa, volvió a perder peso y entonces fue ingresada en un servicio psiquiátrico para adolescentes. Al seguir perdiendo peso (bajó hasta 27 kg.), se la introdujo en un programa conductista, con restricción de todas las 
actividades a menos que comiera las grandes cantidades de comida prescritas.

Cualquier otra actividad placentera, como oír la radio, leer, o realizar manualidades, se le prohibía también si no ganaba peso. Bajo este régimen llegó a $40 \mathrm{~kg}$. en unos cinco meses, resultado que fue descrito en el informe del hospital como un gran éxito. Como siempre, su actividad, su alimentación y sus patrones de sueño quedaron desorganizados durante este programa. Comenzó a pasar días enteros en la cama en un esfuerzo para controlar su alimentación compulsiva, lo que la llevó a estar continuamente durmiendo.

Después de ser dada de alta en la unidad de adolescentes, cerca de unos dos años antes de que nos consultaran, se la introdujo en una terapia familiar, en lugar de una terapia individual. La paciente reaccionó ante esto con un intento serio de suicidio, que obligó a hospitalizarla y a una larga medicación. Durante este tiempo aumento a $45 \mathrm{~kg}$. También le comenzó la menstruación espontáneamente. Logró bajar su peso otra vez provocándose vómitos después de realizar comidas desenfrenadas. Al terminar High Scholl siguió viviendo en casa de sus padres, incapaz de realizar ninguna actividad regularmente, durmiendo todo el dia, empleando las noches en comidas desenfrenadas y vómitos, atormentándose continuamente por el miedo a engordar.

\section{COMENTARIO}

Todas estas pacientes habian ganado peso bajo un programa de modificación de conducta, pero lo habian perdido desde que se les habia dado el alta, o se habían escapado del hospital. Clínicamente se sabe desde hace tiempo que los pacientes de anorexia nerviosa, mientras están hospitalizados, ganan peso bajo la presión de la persuasión, la fuerza, o las amenazas. Deberían "comer por sí mismos al salir del hospital», pero la mayo- ría perdian el peso ganado en muy poco tiempo. Se sabe que sólo se obtiene una verdadera mejoria cuando la ganancia de peso es sólo un aspecto de un programa de tratamiento que integre otros aspectos, con corrección de los problemas familiares e individuales subyacentes ( 3 , p. $309 ; 10)$.

Los defensores de la modificación de conducta pretenden que este enfoque es superior a otros métodos porque el peso ganado se obtiene rápidamente y también porque es infalible. En un marco de carencia afectiva, la obtención de peso es la única posibilidad (6). Esta afirmación es probablemente correcta, pero contiene la explicación de por qué este método provoca perjuicios psicológicos. Hay gran aumento de la confusión interna de los pacientes que sienten que han sido inducidos fraudulentamente a renunciar al control sobre sus cuerpos y sus vidas. Incluso con una ayuda psicoterapéutica pertinente, sólo pueden desarrollar un sentido interno de control psíquico, competencia e identidad, de forma muy lenta.

\section{SENTIMIENTO DE INUTILIDAD VIVIDO POR LOS PACIENTES}

El sentimiento de profunda inutilidad es una de las raíces del problema en el desarrollo de la anorexia nerviosa. La lucha por lograr una iniciativa propia y una identidad, es el problema principal después de una infancia con una obediencia de robot ( 3 , p. 250). Las consecuencias psicológicas de la modificación de conducta son desastrosas porque los pacientes se sienten atrapados en un dilema abrumador, se sienten forzados a escoger entre dos males igualmente inaceptables. Asi, sin soporte psicológico ni confianza en un mejor autoconocimiento, este método socava los últimos rastros de autoestima y destruye la esperanza crucial de obtener una autonomía y autodeterminación.

Los pacientes anoréticos se han queja- 
do siempre de sus experiencias en el hospital, pero no con el mismo cinismo agrio ni con el mismo sentimiento de haber sido profundamente traicionados, expuesto por aquellos que han sido sometidos a la modificación de conducta. Mis pacientes habian experimentado, por igual, el programa como una coerción brutal, por medio de la cual fueron reducidos a un profundo desamparo; por lo que toda la autoconfianza que deberian haber adquirido en una terapia individual, era anulada. A pesar de haber aumentado de peso, consideraron su experiencia como antiterapéutica y hablaban de ella, con verdadera angustia, como un sufrimiento no superado. Lo resumiría en esto: «No hace falta una gran ingeniosidad para inventar un plan para obligar a alguien a engordar. Si pones las cosas lo suficientemente desagradables, aumentarán de peso para escapar a la situación... Yo dejé el hospital deprimida con mi cuerpo, desorientada, y me sentí aparte completamente.»

Con no poca frecuencia, aparecen nuevos síntomas, tales como una alimentación desenfrenada (binge), y vómitos (caso 2), y estos sintomas combinados con alteraciones en el dormir (caso 3). Los pacientes, al igual que sus padres, atribuyeron la desorganización de la conducta a la influencia perjudicial del programa conductista. El daño es incluso mayor en aquellas pacientes «colaboradoras» que no se defienden; simplemente renuncian a luchar y sucumben al sentimiento de desamparo. El resultado puede ser una depresión suicida (caso 1). Este tipo de síntomas aparecieron mientras las pacientes estaban hospitalizadas, y estas pacientes consideraban el hecho de que su situación psicológica no fuera atendida como una crueldad especial del programa.

La terapia de conducta está recomendada como un método que puede ser practicado por personal no médico. La falta de atención al deterioro del cuadro total puede estar relacionada con este hecho: el método es practicado por gente que no posee el conocimiento, ni la comprensión, de esta compleja enfermedad.

\section{PELIGROS POSIBLES}

Los defensores de este método no desconocen los posibles peligros, un ejemplo dramático apareció en el primer informe de Blinder y al. (4), era el caso de una joven que habia ganado $9 \mathrm{~kg}$. durante el tratamiento de modificación de conducta, y que se suicidó unas semanas después de que le dieran el alta. Antiguos autores consideraban la autoemaciación como una expresión de intentos suicidas; estudios modernos -la han revelado como lo opuesto, un esfuerzo de autoaserción. En los pacientes bajo mi observación, el aumento forzado de peso exacerbaba la sensación de desamparo y provocaba una preocupación suicida. En un cuidadoso seguimiento de 94 pacientes, Theander (11) observó que el suicidio solo se producia después de que el peso hubiera alcanzado o superado un nivel normal.

Lo que sustenta el argumento de la necesidad del aumento de peso es el hecho de que la anorexia nerviosa es un estado con una tasa bastante alta de mortalidad, como si esto sirviera de protección contra este destino. Una cuidadosa. evaluación de las historias de aquellos casos con desenlace fatal demuestra trágicamente que el considerar únicamente el aumento de peso es un signo equívoco de recuperación. Ya se ha publicado previamente detalles acerca de 5 pacientes anoréxicas que murieron en ese estado (12). Un joven de catorce años murió de una infección aguda que se le produjo en los primeros meses de la enfermedad, cuando su peso era todavía bastante alto. En los otros 4 pacientes, el peso había sido restaurado tempranamente en el curso de la enfermedad; se les consideró recuperados; aunque los problemas subyacentes no habían sido resueltos, la muerte sobrevino durante una recaida, normalmente varios años después. Desde entonces he evaluado 2 casos más con historias similares. 


\section{CONCLUSION}

Se puede argumentar que los pacientes que he descrito sólo representan unos pocos ejemplos con evoluciones inusuales. Esto es verdad hasta cierto punto, ya que la anorexia nerviosa es un estado raro e infrecuente a pesar de que se vea con mayor frecuencia en la actualidad. Los 3 casos vistos por un observador en un período de 6 meses representa una incidencia elevada. 6 pacientes adicionales han sido estudiados en detalle; cada uno de ellos conocía una o más personas anoréxicas que habían sufrido lo mismo, sumándose a esto muchas cartas con informaciones sobre resultados igualmente pobres.

Hasta que los terapeutas de conducta no informen sobre seguimientos de períodos suficientemente largos, mis hallazgos representan los únicos datos reales sobre los estudios a largo plazo de este método. Posiblemente existan pacientes con casos leves de anorexia nerviosa para los cuales el incremento de peso puede significar el fin de sus dificultades. La literatura ha revelado siempre una contradicción peculiar: los informes de especialistas médicos muestran mejores resultados que los de los psiquiatras. Esto refleja simplemente el hecho de que los psiquiatras sólo ven aquellos casos (pacientes) en los que el tratamiento médico ha fallado.

Puede que la modificación de conducta represente una buena ayuda en el tratamiento de los casos leves; sin embargo, no deja de ser una amenaza para los pacientes con trastornos psicológicos profundos.

En los casos en los que existen trastornos manifiestos de personalidad, está claramente contraindicado el empleo de la modificación de conducta en el tratamiento de la anorexia nerviosa.

\section{Referencias}

1. Selvini, M. P.: «Anorexia Nervosa». London, Chaucer Publishing Co, 1974. (originally in Italian: L'Anoressia Mentale, Milan, Italy, Feltrinelli 1963).

2. BrUCH, H.: «Psychotherapy in primary anorexia nervosam. J. Nerv. Ment. Dis 150: 51-67, 1970.

3. BruCH, H.: «Eating Disorders: Obesity». Anorexia Nervosa and the Person Within. New York, Basic Books, Inc. Publishers, 1973.

4. Blinder, B. J., Freemin, D. M. A., Stunkard, A. J.: «Behavior therapy of anorexia nervosa: Effectiveness of activity as a reinforcer of weight gain. Am». J. Psycbiatry 126-77-82, 1970.

5. Stunkard, A. J.: «New therapies for the eating disordersm. Arcb. Gen. Psycbiatry 26: 391-398, 1972.

6. AgRas, W. S., Barlow, D. H., ChapiN, H. N. et al: «Behavior modification of anorexia nervosa». Arch. Gen. Psycbiatry. 30: 279-286, 1974.

7. «Behavior analysis and behavior therapy", editorial JAMA 220: 852-853, 1972.

8. HuSSEY, H. H.: «Anorexia nervosa: Treatment by behavior modification». JAMA 228: 344, 1974.

9. Feinstein, S. C.: «Anorexia nervosa». JAMA 228: 1230, 1974.

10. Silverman, J. A.: «Anorexia nervosa: Clinical observations in a successful treatment plan». J. Pediatr.» 84:68-73, 1974.

11. Theander, S.: «Anorexia nervosa: Apsychiatrie investigation of 94 female patients». Acta Psycbiatr. Scand., suppl. 214, 1970.

12. BruCH, H.: «Death in anorexia nervosa». Psycboson Med. 33: 135-144, 1971. 